\title{
Ultrafast Deactivation of Excitation Energy in Rutin and Quercetin via Electron and Proton Transfers.
}

\author{
Bondarev SL*, Tikhomirov SA, Knyukshto VN, Buganov OV, Raichenok TF \\ B.I. Stepanov Institute of Physics, National Academy of Sciences of Belarus, Minsk, Nezavisimosti Av. 70, Belarus
}

\begin{abstract}
Using femtosecond spectroscopy and steady-state luminescence methods, the mechanisms of very fast non-radiative deactivation $\left(k_{n r} \sim 5 \times 10^{11} c^{-1}\right)$ of the electronic excitation energy at room temperature in organic and buffer solutions of well-known natural antioxidants rutin and quercetin have been studied.
\end{abstract}

Keywords: Polyphenolic, Blood pressure, Antioxidents, Intensity.

Quercetin (QU) and rutin (RU) belong to the flavonoids (FV). They are a large class of polyphenolic natural pigments found in all land-based green plants [1]. The molecular structure shown on Figure 1, contains two benzene rings (A and $\mathrm{B}$ ) linked through a heterocyclic pyran ring $\mathrm{C}$ (with a double bond) and disaccharide rutinose (RU) or $\mathrm{H}$ (QU) as substitutes in 3-position (R). Among a large number of FV, RU and QU are remarkable for their expressive properties to antagonize the increasing in capillary fragility, to reduce high blood pressure, to serve as antioxidants, etc.

RU and QU are biosynthesized from the amino acid phenylalanine under enzymatic control activated by UV solar radiation [2,3]. The main part of solar radiation in the region $320-400 \mathrm{~nm}$ (UV-A) reaches the Earth's surface participating in damaging photochemical reactions in plants and human skin. It is believed that FV play an important role in the protection of UV-sensitive molecules in living tissues owing to their strong absorption in UV-A region. Accepting high intensity sunny light, the FV has a possibility to save their primary biochemical and spectral properties. Knowing this natural quality of such biological active pigments, we solved to investigate the mechanisms of the effective deactivation processes of electronic excitation energy for rutin and quercetin in organic and buffer solutions at room temperature and $77 \mathrm{~K}$.

Absorption spectra of RU and QU in methanol consist of three vibronic bands with maxima at $357 \mathrm{~nm}$ (band I), $257 \mathrm{~nm}$ (II) and $205 \mathrm{~nm}$ (III). The weak fluorescence spectrum for RU in<smiles>[R]Oc1c(-c2ccc(O)c(O)c2)oc2cc(O)cc(O)c2c1=O</smiles>

Figure 1: Chemical structures of rutin and quercetin. methanol with a maximum at $415 \mathrm{~nm}$ under $\lambda_{\text {exc }}=340 \mathrm{~nm}$ was observed at $293 \mathrm{~K}$ (Figure 2). The measured fluorescence quantum yield $\Phi_{f l}$ was equal to $2.0 \times 10^{-4}$. For QU in acetonitrile the value of $\Phi_{f l}$ is approximately the same. The radiative and non-radiative decay rates of RU are $7.5 \times 10^{7} \mathrm{~s}^{-1}$ and $3.7 \times 10^{11}$ $\mathrm{S}^{-1}$, respectively.

Large increase of rutin's fluorescence intensity is observed at $77 \mathrm{~K}$. The emission spectrum of RU in ethanol consists of two bands (Figure 3): an intensive short-wavelength band at $415 \mathrm{~nm}$ and a weak band at $540 \mathrm{~nm}$. The former emission band belongs to the normal Stokes' shift fluorescence and the second one belongs to the phosphorescence. In the case of QU, there are also 2 emission bands at $293 \mathrm{~K}$ and $77 \mathrm{~K}$ which are characterized as a fluorescence of solvated molecules of QU at $420 \mathrm{~nm}$ and photo-tautomer with intramolecular proton transfer in excited state at $545 \mathrm{~nm}$ [4].

The femtosecond measurements of RU and QU solutions at room temperature allowed revealing the transient absorption spectra at $\lambda_{\max } \sim 460 \mathrm{~nm}$ in all used solvents (Figure 4 ). The deactivation kinetics in all studied solvents are exponential, and the measured lifetimes are located in the range 10.0-20,0 ps. As seen from Figure 4, the spectra reach the maximum within $1.0 \mathrm{ps}$ and organic vanish absolutely through $100.0 \mathrm{ps}$.

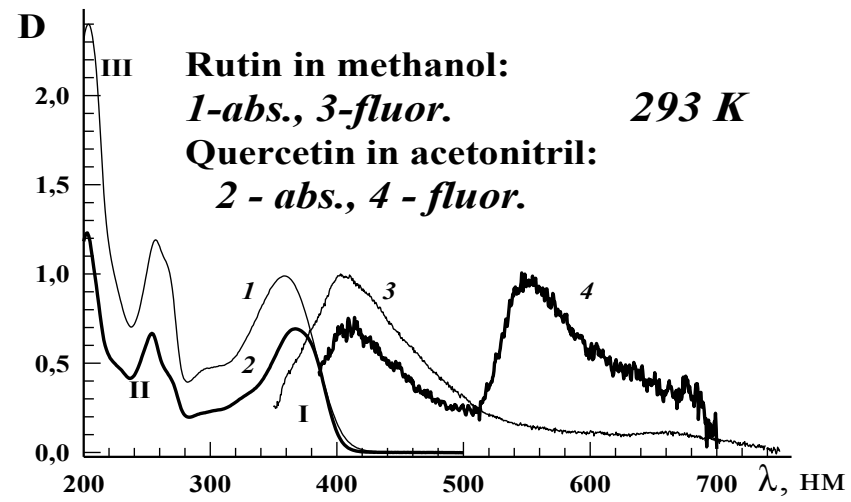

Figure 2. Absorption, fluorescence spectra of $R U$ in methanol. 


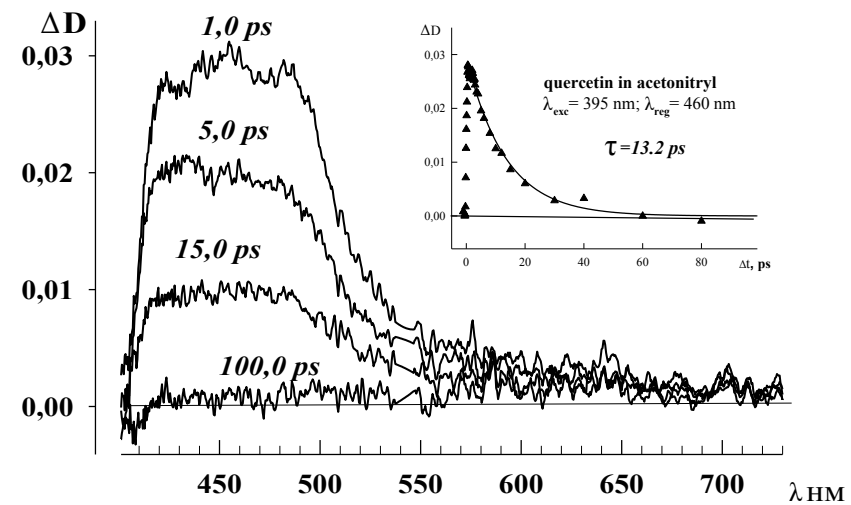

Figure 3. Luminescence spectra of $R U$ (1) and $Q U$ in acetonitrile at $293 K$.

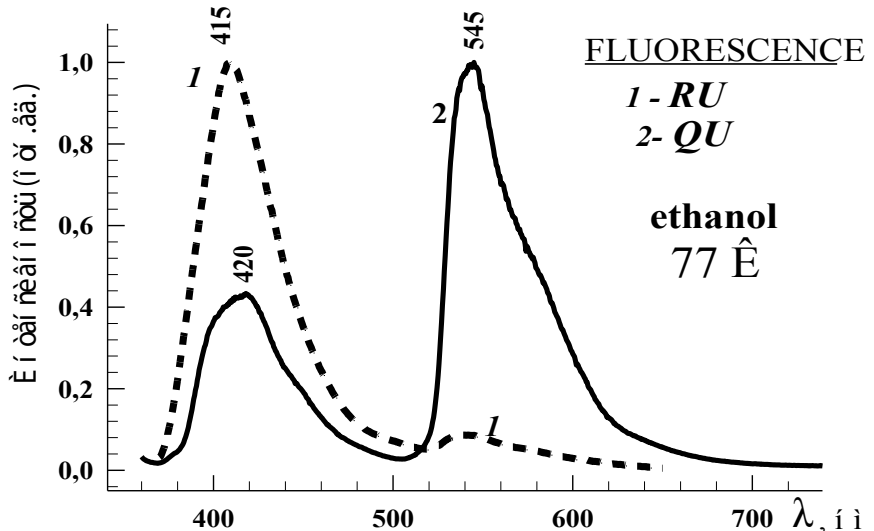

Figure 4. Transient absorption spectra and $Q U(2)$ in ethanol at $77 \mathrm{~K}$ kinetics of $Q U$ in acetonitrile at $293 \mathrm{~K}$.

The triplet-triplet absorption wasn't revealed in all spectral region.

The femtosecond measurements of RU and QU organic solutions at room temperature allowed revealing the transient absorption spectra at $\lambda_{\max } \sim 460 \mathrm{~nm}$ in all used solvents (Figure 4). The deactivation kinetics in all studied solvents are exponential, and the measured lifetimes are located in the range $10.0-20.0 \mathrm{ps}$. As seen from Figure 4, the spectra reach the maximum within $1,0 \mathrm{ps}$ and vanish absolutely through $100.0 \mathrm{ps}$. The triplet-triplet absorption wasn't revealed in all studied spectral region.

From these data along with very low fluorescence quantum yield at room temperature we can propose the mechanisms of very effective non-emission deactivation in RU and QU. The differences in structural and spectral properties for these two flavonoids have determined the differences in their nonemission channels of electronic excitation energy deactivation. The first difference is in large spectral shift of the low excited state in QU as compare with RU: $24100 \mathrm{~cm}^{-1}(415 \mathrm{~nm})$ for RU and $18500 \mathrm{~cm}^{-1}(540 \mathrm{~nm})$ for QU. However, the form and kinetic of transient absorption spectra at room temperature for RU and QU are practically the same. This fact means that part of RU and QU molecules deactivate the same way. This way is determined by the solvates of RU and QU molecules which are organized in the ground state. The another non-solvated part of RU molecules must possess a very small probability of direct internal conversion due to very large energy gap $\Delta \mathrm{E}\left(\mathrm{S}_{1}, \mathrm{~S}_{0}\right) \approx 27800 \mathrm{~cm}^{-1}$. However, it is observed an opposite process of high rising of internal conversion. Our explanation of that unusual behavior is consists in the new transient excited state appearing. That transient state has the charge (electron) transfer (CT) character. The Frank-Condon excited states of RU and QU are characterized by high dipole moments 46.6 $\times 10^{-30} \mathrm{C} \mathrm{m}$ and $52.8 \times 10^{-30} \mathrm{C} \mathrm{m}$, respectively. The adiabatic potential energy of this $\mathrm{CT}$ state is changed in dependence of the rotation angle of B-ring around $2-1$ '-bond. The strong electron transfer is proposed between $\mathrm{B}-$ ring and 4-CO carbonyl group. In the case of QU the formation CT state and localization of the excess electron density at 4-CO carbonyl group promote additionally the intramolecular proton transfer with formation photo-tautomer. Therefore, the main channel of non-emission deactivation of the electronic excitation energy in $\mathrm{RU}$ and $\mathrm{QU}$ is the $\mathrm{S}_{1} \sim \sim>\mathrm{S}_{0}$ internal conversion induced by the CT state.

\section{References}

1. Rothwell JA, Daay AJ, Morgan MRA. Experimental determination of octanole-water partition coefficients of quercetin and related flavonoids, J Agric. Food Chem. 2005;4355-60.

2. Harborne JB, Williams CA. Advances in flavonoid research since 1992, Phytochemistry 2000;481-504.

3. Gaberscik A, Voncina M, Trost $\mathrm{T}$, et al. Growth and production of buckwheat (Fagopyrum esculentum) treated with reduced, ambient, and enhanced UV-B radiation, J Photochem Photobiol. B: Biol. 2000;30-6.

4. Falkovskaia E, Sengupta PK, Kasha M. Photophysical induction of dual fluorescence of quercetin and related hydroxyflavones upon intermolecular H-bonding to solvent matrix. Chem Phys. Lett. 1998;29:109-116.

\section{*Correspondence to:}

Bondarev SL

B.I. Stepanov Institute of Physics

National Academy of Sciences of Belarus

Belarus

Fax: + 37517 284-08-79

E-mail: Stan.Bond.1944@Gmail.Com 